\title{
Keep 'hoping' for rationality: a solution to the backward induction paradox
}

\author{
Alexandru Baltag • Sonja Smets • \\ Jonathan Alexander Zvesper
}

Received: 12 December 2008 / Accepted: 14 April 2009 / Published online: 18 May 2009

(C) The Author(s) 2009. This article is published with open access at Springerlink.com

\begin{abstract}
We formalise a notion of dynamic rationality in terms of a logic of conditional beliefs on (doxastic) plausibility models. Similarly to other epistemic statements (e.g. negations of Moore sentences and of Muddy Children announcements), dynamic rationality changes its meaning after every act of learning, and it may become true after players learn it is false. Applying this to extensive games, we "simulate" the play of a game as a succession of dynamic updates of the original plausibility model: the epistemic situation when a given node is reached can be thought of as the result of a joint act of learning (via public announcements) that the node is reached. We then use the notion of "stable belief", i.e. belief that is preserved during the play of the game, in order to give an epistemic condition for backward induction: rationality and common knowledge of stable belief in rationality. This condition is weaker than Aumann's and compatible with the implicit assumptions (the "epistemic openness of the future") underlying Stalnaker's criticism of Aumann's proof. The "dynamic" nature of our concept of rationality explains why our condition avoids the apparent circularity of the "backward induction paradox": it is consistent to (continue to) believe in a player's rationality after updating with his irrationality.
\end{abstract}

\footnotetext{
A. Baltag

Oxford University Computing Laboratory, University of Oxford, Oxford OX1 3QD, UK

e-mail: baltag@comlab.ox.ac.uk
}

\section{S. Smets}

University of Groningen, Groningen, The Netherlands

e-mail: sonsmets@vub.ac.be

S. Smets

Oxford University, Oxford, UK

\section{J. A. Zvesper $(\varangle)$}

Institute for Logic, Language and Computation, Universiteit van Amsterdam,

Plantage Muidergracht 24, Amsterdam 1018 TV, Netherlands

e-mail: jonathan@illc.uva.nl 
Keywords Backward induction - Dynamic logic · Epistemic logic · Public announcements · Rationality

Aumann has proved that common knowledge of substantive rationality implies the backward induction solution in games of perfect information.

Stalnaker has proved that it does not.

Halpern (2001)

The jury is still out concerning the epistemic conditions for backward induction, the "oldest idea in game theory" (Aumann 1995, p. 635). Aumann (1995) and Stalnaker (1996) take conflicting positions in the debate: the former claims that common "knowledge" of "rationality" in a game of perfect information entails the backward induction solution; the latter that it does not. ${ }^{1}$ Of course there is nothing wrong with any of their relevant formal proofs, but rather, as pointed out by Halpern (2001), there are differences between their interpretations of the notions of knowledge, belief, strategy and rationality. Moreover, as pointed out by Binmore (1987, 1996), Bonanno (1991), Bicchieri (1989), Reny (1992), Brandenburger (2007) and others, the reasoning underlying the backward induction method seems to give rise to a fundamental paradox: in order even to start the reasoning, a player assumes that (common knowledge of, or some form of common belief in) "rationality" holds at all the last decision nodes (and so the obviously irrational leaves are eliminated); but then, in the next reasoning step (going backward along the tree), some of these (last) decision nodes are eliminated, as being incompatible with (common belief in) "rationality"! Hence, the assumption behind the previous reasoning step is now undermined: the reasoning player can now see that if those decision nodes that are now declared "irrational" were ever to be reached, then the only way that this could happen is if (common belief in) "rationality" failed. Hence, she was wrong to assume (common belief in) "rationality" when she was reasoning about the choices made at those last decision nodes. This whole line of arguing seems to undermine itself!

\section{Belief dynamics}

In this paper we use as a foundation the relatively standard and well-understood setting of Conditional Doxastic Logic (CDL, Board 2002; Baltag and Smets 2006, 2008a, b), and its "dynamic" version (obtained by adding to CDL operators for truthful public announcements $[! \varphi] \psi)$ : the logic PAL-CDL, introduced by van Benthem (2007a). In fact, we consider a slight extension of this last setting, namely the logic APAL-CDL, obtained by further adding dynamic operators for arbitrary (truthful) public announcements [!] $\psi$, as in Balbiani et al. (2008). We use this formalism to capture a novel notion of "dynamic rationality" and to investigate its role in decision problems and games. As usual in these discussions, we take a deterministic stance, assuming that the initial state of the world at the beginning of the game already fully determines the future play, and thus the unique outcome, irrespective of the players' (lack of) knowledge of future

\footnotetext{
1 Others agree with Stalnaker in disagreeing with Aumann: for example, Samet (1996) and Reny (1992) also put forwards arguments against Aumann's epistemic characterisation of subgame-perfect equilibrium. Section 7 is devoted to a discussion of related literature.
} 
moves. We do not, however, require that the state of the world determines what would happen, if that state were not the actual state. That is, we do not need to postulate the existence of any "objective counterfactuals". But instead, we only need "subjective counterfactuals": in the initial state, not only the future of the play is specified, but also the players' beliefs about each other, as well as their conditional beliefs, pre-encoding their possible revisions of belief. The players' conditional beliefs express what one may call their "propensities", or "dispositions", to revise their beliefs in particular ways, if given some particular pieces of new information.

Thus at the outset of a game, all is "done", including the future. But all is not necessarily "said". In a deterministic model, as time progresses the only thing that changes are the pictures of the world in the minds of the players: the information states of the players. This is "on-line" learning: while the game is being played, the players learn the played moves, and so they may change their minds about the situation. We can simulate this on-line learning (and its effect on the players' beliefs) via off-line "public announcements": if, before the start of the game, the agents were publicly told that the game will reach some node $u$, then they would be in the same epistemic state as they would have been by (not having any such public announcement but instead) playing the game until node $u$ was reached.

So in this paper we stress the importance of the dynamics of beliefs and rationality during a play of an extensive game, and we use dynamic operators in order to simulate the play of the game. Since we focus on games of perfect information, we only need public announcements to simulate the moves of the game. The idea of adding modalities for public announcements to epistemic logic was introduced and developed in Plaza (1989) and Gerbrandy and Groeneveld (1997). Dynamic epistemic logic (Baltag et al. 1999) provides for much richer dynamic modalities than just public announcements, capturing the effects of more complex and more "private" forms of learning. We think these could be applied to the case of games with imperfect information. However, for simplicity, we leave these developments for future work and consider for now only perfect information, and so only public announcements.

\section{Games}

Using the terminology of Brandenburger (2007), ours is a belief-based approach to game theory (in the same category as the work of Battigalli and Siniscalchi (1999, 2002)), in contrast to the knowledge-based approach of Aumann (1995) and others. According to the belief-based approach, only observables are knowable. Unobservables are subject to belief, not knowledge. In particular, other players' strategies are unobservables, and only moves are observables (Brandenburger 2007, p. 489). This means that we take the players' beliefs (including conditional beliefs) as basic, instead of their knowledge. However, there is a notion of knowledge that naturally arises in this context: the "irrevocable knowledge", consisting of the beliefs that are absolutely unrevisable, i.e. believed under any conditions. This notion of knowledge is meant to apply only to the players' "hard information", obtained by observation or by undoubtable evidence. This is a much stronger condition than "certain belief" (subjective probability 1) or even "true belief", and as a result it may happen that very few things 
are "known" in this sense. One of the things we assume to be irrevocably known is the structure of the game: the possible outcomes, the players' preferences etc; also, in a game of perfect information, the played moves are observed, and thus known, after they are played; finally, another thing irrevocably known to a player is her own beliefs: by introspection, she knows what she believes and what not. Besides this, we do not assume much else to be known, although our general setting is in principle consistent with (common) knowledge of all the players' beliefs, their strategies, their rationality etc.

One thing we do not assume as known is the future of the game: no outcomes that are consistent with the structure of the game are to be excluded at the outset of the game. In fact, we make the opposite assumption: that it is common knowledge that nobody knows the future, i.e. nobody knows that some outcome will not be reached. This "open future" assumption seems to contradict common knowledge of rationality; but in fact, it is consistent with it, if by rationality we only mean "rational planning", leaving open the possibility that players may make mistakes or may change their minds. The players may certainly believe their rational plans will be faithfully carried out, but they have no way to "know" this in advance. We think of our "open future" assumption as being a realistic one, and moreover one that embodies the agents' "freedom of choice", as well as the "possibility of error", that underly a correct notion of rationality. An agent's rationality can be assessed only if she is given some options to freely choose from. There are certainly cases in which the future can be known, e.g. when it is determined by a known natural law. But it is an essential feature of rational agents that their own choices are not known to them to be thus determined; or else, they would have no real choices, and thus no rational choice. Any natural determinism is assumed to be absorbed in the definition of the game structure, which does pose absolute limits to choices. In a sense, this simply makes precise the meaning of our "knowledge" as "hard information", and makes a strict delimitation between the past and the future choices, delimitation necessary to avoid the various paradoxes and vicious circles that plague the notions of rational decision and freedom of choice: the agents may have "hard information" about the past and the present, but not about their own future free choices (although they may have "soft" information, i.e. "certain" beliefs, with probability 1 , about their future choices).

\section{Dynamic rationality}

Maybe the most important original feature of our paper is our notion of "dynamic" rationality, which takes into account the dynamics of beliefs, as well as the dynamics of knowledge. On the one hand, following Stalnaker, Reny, Battigalli and Siniscalchi etc. (and in contrast with Aumann), we assess the rationality of a player's move at a node against the beliefs held at the moment when the node is reached. On the other hand, we incorporate the above-mentioned epistemic limitation to rationality: the rationality of an agent's move only makes sense when that move is not already known (in an irrevocable manner) to her. Players cannot be held responsible for moves that they cannot choose or change any more (including their own past moves). Since the players' knowledge increases during a game of perfect information, their set of available 
options decreases: passed options/nodes, or nodes that were by-passed, cannot be the objects of choice any more. As a result, our notion of rationality is future-oriented: at any stage of the game, whether or not an agent is dynamically rational at that stage depends only on her current and future moves. So a player can be rational now even if in the past she has made some "irrational" moves. In effect, performing such an irrational move in a game of perfect information is equivalent to a public announcement that "the player is (currently) not rational" (at the moment of moving). ${ }^{2}$ All the players jointly learn this "fact" (as a piece of "hard' information), but the "fact" itself may vanish after being learnt: while previously "irrational" (since about to make a "wrong' move), the player may "become rational" after the wrong move (simply because, for all the decisions that she can still make after that, she chooses the 'right' moves). So the truth-value of the sentence "player $i$ is (dynamically) rational" may change after a move by player $i$. The way this is captured and explained in our formal setting is original and interesting in itself: the meaning of our "rationality" changes in time, due to the change of beliefs and of the known set of options. This is because the "rationality of an agent" is an epistemic-doxastic concept, so it is obviously affected by any changes in the information possessed by that agent (including the changes induced by the agent's own moves). In our Dynamic-Epistemic Logic setting, this is a natural and perfectly standard feature, an immediate consequence of the epistemic definition of rationality: in general, epistemic sentences do not necessarily preserve their truth value after they are "learnt". Epistemic logicians are already familiar with this phenomenon, e.g. the examples of Moore sentences (Moore 1942) and of the repeated public announcements of "ignorance" in the Muddy Children Scenario (Fagin et al. 1995).

Our concept of dynamic rationality, developed on purely a priori grounds, solves in one move the "BI-paradox": the first reasoning step in the backward-induction argument (dealing with the last decision nodes of the game) is not undermined by the result of the second reasoning step, since the notion of "rationality" assumed in the first step is not the same as the "rationality" disproved in the second step! The second step only shows that some counterfactual nodes cannot be reached by rational play, and thus it implies that some agent must have been irrational (or must have had some doubts about the others' rationality, or must have made some "mistake") before such an "irrational" node was reached; but this doesn't contradict in any way the assumption that the agents will be rational at that node (and further in the future).

\section{Stability}

Dynamics cannot really be understood without its correlative: invariance under change. Certain truths, or beliefs, stay true when everything else changes. We have already encountered an "absolute" form of invariance: "irrevocable knowledge", i.e. belief that is invariant under any possible information change. Now, we need a second, weaker form of invariance: "stability". A truth, or a belief, is stable if it remains true, or

\footnotetext{
2 Technically, this claim is correct only for binary games, in which at any node there are only two possible moves; but a weak version of this claim holds in general.
} 
continues to be believed, after any (joint) learning of "hard" information (via some truthful public announcement). In fact, in the case of an "ontic" (non-doxastic) fact $p$, Stalnaker's favourite notion of "knowledge" of $p$ (Stalnaker 1996, 2006) (a modal formalisation of Lehrer and Klein's "defeasibility theory of knowledge"), also called "safe belief" in Baltag and Smets (2008b), corresponds precisely to stable belief in $p$. (But note that the two notions differ when applied to a doxastic-epistemic property, such as "rationality"!) Stability can be or not a property of a belief or a common belief: a proposition $P$ is a "stable (common) belief" if the fact that $P$ is (common) belief is a stable truth, i.e. $P$ continues to be (common) belief after any (joint) learning of "hard" information.

What is required for achieving the backward induction outcome is stable belief in dynamic rationality, as a default condition (i.e. commonly known to hold for all agents). In some contexts, we can think of this condition as expressing an "optimistic" belief-revision policy about the opponents' potential for rationality: the players "keep hoping for rationality" with respect to everybody's current and future play, despite any past irrational moves. Of course, whether or not the words "hope" and “optimism" are appropriate depends on the players' payoffs: e.g. in common interest games (in which all players' payoffs are identical at all nodes), it indeed makes sense to talk about "hoping" for opponents' rationality; while in other games, it may be more appropriate to talk about "persistent cautiousness" and a "pessimistic" revision policy.

We can now give an informal statement of the main theorem of this paper:

Common knowledge of (the game structure, of "open future" and of) stable $\left(\right.$ common $\left.^{3}\right)$ belief in dynamic rationality entails common belief in the backward induction outcome.

\section{Overview of the paper}

To formalise stability and "stable common belief", we introduce in the next section Conditional Doxastic Logic CDL and its dynamic version APAL-CDL. Section 2 recalls the definition of extensive games and shows how to build models of those games in which the structure of the game is common knowledge, in our strong sense of "knowledge". In Sect. 3 we define "rationality" and "rational play", starting from more general decision-theoretic considerations, and arriving at a definition of dynamic rationality in extensive (aka "dynamic") games, which is in some sense a special case of the more general notion. Section 4 gives a formal statement of our main results, to whose proofs Sect. 5 is devoted. In Sect. 6 we consider a weaker condition that ensures the backward induction outcome, and is based on what we call stable true belief. Finally, Sect. 7 discusses connections between our work and some of the existing literature on the epistemic foundations of backward induction.

\footnotetext{
3 Adding the word "common" to this condition doesn't make a difference: common knowledge that everybody has a stable belief in $P$ is the same as common knowledge of common safe belief in $P$.
} 


\section{Conditional doxastic logic and its dynamic extensions}

CDL models, also called "plausibility models" are essentially the "belief revision structures" in Board (2002), simplified by incorporating structurally the assumption of Full Introspection of Beliefs (which allows us to use binary plausibility relations on worlds for each agent, instead of ternary relations). But since we will also want to talk about the actual change under the effects of actions, like moves in a game, rather than just the static notion that is in effect captured by Board's models, we will enrich the language of CDL with model-changing dynamic operators for "public announcements", in the spirit of Dynamic Epistemic Logic (cf. Baltag et al. 1999; van Benthem 2007a, b).

The models are "possible worlds" models, where the worlds will usually be called states. Grove (1988) showed that the AGM postulates (Alchourrón et al. 1985) for rational belief change are equivalent to the existence of a suitable pre-order over the state space. ${ }^{4}$ The intended interpretation of the pre-order $\leq_{i}$ of some agent $i$ is the following: $s \leq_{i} t$ means that, in the event $\{s, t\}, i$ considers $s$ at least as plausible as $t$.

In interactive situations, where there are several players, each player $i$ has a doxastic pre-order $\leq_{i}$. In addition to having different beliefs, any two players might have different knowledge. We follow the mainstream in game theory since Aumann and model interactive knowledge using a partitional structure. However, as in Board (2002), we will derive $i$ 's partition from $i$ 's pre-order $\leq_{i}$. Let us be more precise: fix a set $S$ and a relation $\leq_{i} \subseteq S \times S$; then we define the comparability class of $s \in S$ for $\leq_{i}$ to be the set $[s]_{i}=\left\{t \in S \mid s \leq_{i} t\right.$ or $\left.t \leq_{i} s\right\}$ of states $\leq_{i}$-comparable to $s$. Now we want the set of comparability classes to form a partition of $S$, so we will define a plausibility frame to be a sequence $\left(S, \leq_{i}\right)_{i \in N}$ in which $S$ is a non-empty set of states, and each $\leq_{i}$ a pre-order on $S$ such that for each $s \in S$, the restriction of $\leq_{i}$ to $[s]_{i}$ is a "complete" (i.e. "total" or "connected") pre-order.

Fact 1.1 In any plausibility frame, $\left\{[s]_{i} \mid s \in S\right\}$ forms a partition of $S$. We will interpret this as the information partition for player $i$ (in the sense of "hard" information, to be explained below).

So we can define player $i$ 's knowledge operator in the standard way, putting for any "proposition" $P \subseteq S$ :

$$
K_{i} P:=\left\{s \in S \mid[s]_{i} \subseteq P\right\}
$$

As explained below, this captures a notion of indefeasible, absolutely unrevisable knowledge. But we also want a notion of belief $B$, describing "soft" information, which might be subject to revision. So we want conditional belief operators $B^{P}$, in order to capture the revised beliefs given some new information $P$. If $S$ is finite, let $\min _{\leq_{i}}(P)$ denote the $\leq_{i}$-minimal $P$ elements $\left\{s \in P \mid \forall t \in P, s \leq_{i} t\right\}$. So $\min _{\leq_{i}}(P)$ denotes the set of states which $i$ considers most plausible given $P$. Then $\min _{\leq i}\left(P \cap[s]_{i}\right)$ denotes the set of that states which $i$ considers most plausible given

\footnotetext{
4 A pre-order is any reflexive transitive relation. In Grove's representation theorem the pre-order must also be total and converse-well-founded.
} 
both $P$ and $i$ 's knowledge at state $s$. Thus we define player $i$ 's conditional belief operator as:

$$
B_{i}^{Q} P:=\left\{s \in S \mid \min _{\leq i}\left(Q \cap[s]_{i}\right) \subseteq P\right\} .
$$

There is a standard way to extend this definition to total pre-orders on infinite sets of states, but we skip here the details, since we are mainly concerned with finite models. $B_{i}^{Q} P$ is the event that agent $i$ believes $P$ conditional on $Q$. Conditional belief should be read carefully: $B_{i}^{Q} P$ does not mean that after learning that $Q, i$ will believe $P$; rather it means that after learning $Q, i$ will believe that $P$ was the case before the learning. This is a subtle but important point: the conditional belief operators do not directly capture the dynamics of belief, but rather as van Benthem (2007a) puts it, they 'pre-encode' it. We refer to van Benthem (2007a) and Baltag and Smets (2008b) for more discussion. The usual notion of (non-conditional) belief can be defined as a special case of this, by putting $B_{i} P:=B_{i}^{S} P$. The notions of common knowledge $C k P$ and common belief $C b P$ are defined in the usual way: first, one introduces general knowledge EkP $:=\bigcap_{i} K_{i} P$ and general belief EbP $:=\bigcap_{i} B_{i} P$, then one can define $C k P:=\bigcap_{n}(E k)^{n} P$ and $C b P:=\bigcap_{n}(E b)^{n} P$.

It will be useful to associate with the states $S$ some non-epistemic content; for this we use a valuation function. Assume given some finite set $\Phi$ of symbols, called basic (or atomic) sentences, and meant to describe ontic (non-epistemic, non-doxastic) "facts" about the (current state of the) world. A valuation on $\Phi$ is a function $V$ that associates with each $p \in \Phi$ a set $V(p) \subseteq S: V$ specifies at which states $p$ is true. A plausibility model for (a given set of atomic sentences) $\Phi$ is a plausibility frame equipped with a valuation on $\Phi$.

\subsection{Interpretation: 'hard' and 'soft' information}

Information can come in different flavours. An essential distinction, due to van Benthem (2007a), is between 'hard' and 'soft' information. Hard information is absolutely "indefeasible", i.e. unrevisable. Once acquired, a piece of 'hard' information forms the basis of the strongest possible kind of knowledge, one which might be called irrevocable knowledge and is denoted by $K_{i}$. For instance, the principle of Introspection of Beliefs states that (introspective) agents possess 'hard' information about their own beliefs: they know, in an absolute, irrevocable sense, what they believe and what not. Soft information, on the other hand, may in principle be defeated (even if it happens to be correct). An agent usually possesses only soft information about other agents' beliefs or states of mind: she may have beliefs about the others' states of mind, she may even be said to have a kind of 'knowledge' of them, but this 'knowledge' is defeasible: in principle, it could be revised, for instance if the agent were given more information, or if she receives misinformation.

For a more relevant, game-theoretic example, consider extensive games of perfect information: in this context, it is typically assumed (although usually only in an implicit manner) that, at any given moment, both the structure of the game and the players' past moves are 'hard' information; e.g. once a move is played, all players 
know, in an absolute, irrevocable sense, that it was played. Moreover, past moves (as well as the structure of the game) are common knowledge (in the same absolute sense of knowledge). In contrast, a player's 'knowledge' of other players' rationality, and even a player's 'knowledge' of her own future move at some node that is not yet reached, are not of the same degree of certainty: in principle, they might have to be revised; for instance, the player might make a mistake, and fail to play according to her plan; or the others might in fact play "irrationally", forcing her to revise her 'knowledge' of their rationality. So this kind of defeasible knowledge should better be called 'belief', and is based on players' "soft" information. ${ }^{5}$

In the 'static' setting of plausibility models given above, soft information is captured by the "belief" operator $B_{i}$. As already mentioned, this is defeasible, i.e. revisable, the revised beliefs after receiving some new information $\varphi$ being pre-encoded in the conditional operator $B_{i}^{\varphi}$. Hard information is captured by the "knowledge" operator $K_{i}$; indeed, this is an absolutely unrevisable form of belief, one which can never be defeated, and whose negation can never be accepted as truthful information. This is witnessed by the following valid identities:

$$
K_{i} P=\bigcap_{Q \subseteq S} B_{i}^{Q} P=B_{i}^{\neg P} \emptyset .
$$

\subsection{Special case: conditional probabilistic systems}

If, for each player $i$, we are given a conditional probabilistic system à la Rényi (1955) over a common set of states $S$ (or if alternatively we are given a lexicographic probability system in the sense of Blume et al. 1991), we can define subjective conditional probabilities $\operatorname{Prob}_{i}(P \mid Q)$ even for events of zero probability. When $S$ is finite and the system is discrete (i.e., $\operatorname{Prob}(P \mid Q)$ is defined for all non-empty events $Q$ ), we can use this to define conditional belief operators for arbitrary events, by putting $B_{i}^{Q} P:=\left\{s \in S: \operatorname{Prob}_{i}(P \mid Q)=1\right\}$. It is easy to see that these are special cases of finite plausibility frames, by putting: $s \leq_{i} t$ iff $\operatorname{Prob}_{i}(\{s\} \mid\{s, t\}) \neq 0$. Moreover, the notion of conditional belief defined in terms of the plausibility relation is the same as the one defined probabilistically as above.

\subsection{Dynamics and information: 'hard' public announcements}

Dynamic epistemic logic is concerned with the "origins" of hard and soft information: the "epistemic actions" that can appropriately inform an agent. In this paper, we will focus on the simplest case of hard-information-producing actions: public announcements. These actions model the simultaneous joint learning of some 'hard' piece of information by a group of agents; this type of learning event is perfectly "transparent"

\footnotetext{
5 By looking at the above probabilistic interpretation, one can see that the fact that an event or proposition has (subjective) probability 1 corresponds only to the agent having "soft" information (i.e. believing the event). "Hard" information corresponds to the proposition being true in all the states in the agent's information cell.
} 
to everybody: there is nothing hidden, private or doubtful about it. But dynamic epistemic logic (Baltag et al. 1999) also deals with other, more complex, less transparent and more private, forms of learning and communication.

Given a plausibility model $\mathcal{M}=\left(S, \leq_{i}, V\right)_{i \in N}$ and a "proposition" $P \subseteq S$, the updated model $\mathcal{M} \uparrow P$ produced by a public announcement of $P$ is given by relativisation: $\left(P, \leq_{i} \uparrow P, V \uparrow P\right)$, where $\leq \uparrow P$ is the restriction of $\leq$ to $P$ and $(V \uparrow P)(p)=V(p) \cap P$. Notice that public announcements can change the knowledge and the beliefs of the players. So far we have, for readability, been writing events without explicitly writing the frame or model in question. However, since we are now talking about model-changing operations it is useful to be more precise; for this we will adopt a modal logical notation.

\subsection{APAL-CDL: language and semantics}

Our language APAL-CDL is built recursively, in the usual manner, from atomic sentences in $\Phi$, using the Boolean connectives $\neg \varphi, \varphi \wedge \psi, \varphi \vee \psi$ and $\varphi \Longrightarrow \psi$, the epistemic operators $K_{i} \varphi, B_{i}^{\varphi} \psi, C k \varphi$ and $C b \varphi$ and the dynamic modalities [! $\left.\varphi\right] \psi$ and $[!] \varphi$. (The language $C D L$ of conditional doxastic logic consists only of the formulas of APAL-CDL that can be formed without using the dynamic modalities.)

For any formula $\varphi$ of this language, we write $\llbracket \varphi \rrbracket \mathcal{M}$ for the interpretation of $\varphi$, the event denoted by $\varphi$, in $\mathcal{M}$. We write $\mathcal{M}^{\varphi}$ for the updated model $\mathcal{M} \uparrow \llbracket \varphi \rrbracket_{\mathcal{M}}$ after the public announcement of $\varphi$. The interpretation map is defined recursively: $\llbracket p \rrbracket_{\mathcal{M}}=V(p)$; Boolean operators behave as expected; and the definitions given above of the epistemic operators in terms of events give the interpretation of epistemic formulae. Then the interpretation of the dynamic formulae, which include public announcement modalities $[! \varphi] \psi$, goes as follows:

$$
\llbracket[! \varphi] \psi \rrbracket_{\mathcal{M}}=\left\{s \in S \mid s \in \llbracket \varphi \rrbracket_{\mathcal{M}} \Rightarrow s \in \llbracket \psi \rrbracket_{\mathcal{M}^{\varphi}}\right\}
$$

Thus $[! \varphi] \psi$ means that after any true public announcement of $\varphi, \psi$ holds. The arbitrary (public) announcement modality [!] $\varphi$ is to be read: after every (public) announcement, $\varphi$ holds. Intuitively, this means $\varphi$ is a "stable" truth: not only it is true, but it continues to stay true when any new (true) information is (jointly) learned (by all the players). There are some subtleties here: do we require that the new information/announcement be expressible in the language for example? This is the option taken in Balbiani et al. (2008), where the possible announcements are restricted to epistemic formulas, and a complete axiomatisation is given for this logic. In the context of finite models (as the ones considered here), this definition is actually equivalent to allowing all formulas of our language APAL-CDL as announcements. As a result, we can safely use the following apparently circular definition:

$$
\llbracket[!] \varphi \rrbracket_{\mathcal{M}}=\left\{s \in S \mid \forall \psi s \in \llbracket[! \psi] \varphi \rrbracket_{\mathcal{M}}\right\}
$$

Dynamic epistemic logic captures the "true" dynamics of (higher-level) beliefs after some learning event: in the case of public announcements, the beliefs of an agent $i$ 
after a joint simultaneous learning of a sentence $\varphi$ are fully expressed by the operator $[! \varphi] B_{i}$, obtained by composing the dynamic and doxastic operators. Note that this is not the same as the conditional operator $B_{i}^{\varphi}$, but the two are related via the following "Reduction Law", introduced in van Benthem (2007a):

$$
[! \varphi] B_{i} \psi \Leftrightarrow\left(\varphi \Rightarrow B_{i}^{\varphi}[! \varphi] \psi\right)
$$

This is the precise sense in which the conditional belief operators are said to "preencode" the dynamics of belief.

\subsection{Special case: Bayesian conditioning}

In the case of a conditional probability structure, the update $\mathcal{M} \uparrow P$ by a public announcement $! P$ corresponds to Bayesian update (conditionalisation): the state space is reduced to the event $P$, and the updated probabilities are given by $\operatorname{Prob}_{i}^{\prime}(Q):=$ $\operatorname{Prob}_{i}(Q \mid P)$. So a dynamic modality $[! P] Q$ corresponds to the event that, after conditionalising with $P$, event $Q$ holds. Similarly, the arbitrary announcement modality [!] $P$ is the event that $P$ stably holds, i.e. it holds after conditionalising with any true event.

\section{Models and languages for games}

The notion of extensive game with perfect information is defined as usual (cf. Osborne and Rubinstein 1994): Let $N$ be a set of 'players', and $G$ be a finite tree of 'decision nodes', with terminal nodes (leaves) $\mathcal{O}$ (denoting "possible outcomes"), such that at each non-terminal node $v \in G-\mathcal{O}$, some player $i \in N$ is the decision-maker at $v$. We write $G_{i} \subseteq G$ for the set of nodes at which $i$ is the decision-maker. Add to this a payoff function $h_{i}$ for each player $i$, mapping all the leaves $o \in \mathcal{O}$ into real numbers, and you have an extensive game. We write ' $G$ ' to refer both to the game and to the corresponding set of nodes. We also write $u \rightarrow v$ to mean that $v$ is an immediate successor of $u$, and $u \rightsquigarrow v$ to mean that there is a path from $u$ to $v$. A subgame of a game $G$ is any game $G^{\prime}$, having a subset $G^{\prime} \subseteq G$ as the set of nodes and having the immediate successor relation $\rightarrow^{\prime}$, the set of decision nodes $G_{i}^{\prime}$ and the payoff function $h_{i}^{\prime}$ (for each player $i$ ) being given by restrictions to $G^{\prime}$ of the corresponding components of the game $G$ (e.g. $G_{i}^{\prime}=G_{i} \cap G^{\prime}$ etc). For $v \in G$, we write $G^{v}$ for the subgame of $G$ in which $v$ is the root. A strategy $\sigma_{i}$ for player $i$ in the game $G$ is defined as usually as a function from $G_{i}$ to $G$ such that $v \rightarrow \sigma_{i}(v)$ holds for all $v \in G_{i}$. Similarly, the notions of strategy profile, of the (unique) outcome determined by a strategy profile and of subgame-perfect equilibrium are defined in the standard way (see e.g. Osborne and Rubinstein 1994). Finally, we define as usually a backward induction outcome to be any outcome $o \in \mathcal{O}$ determined by some subgame-perfect equilibrium. We denote by $B I_{G}$ the set of all backward-induction outcomes of the game $G$.

Consider as an example the "centipede" game $G$ (cf. Rosenthal 1981) given in Fig. 1. This is a two-player game for $a$ (Alice) and $b$ (Bob). 
Fig. 1 The "centipede" game $G$

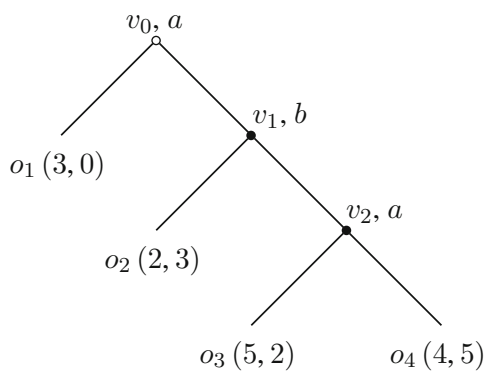

Here, we represent the nodes of the game by dots and the possible moves by arrows. For each non-terminal node, the corresponding dot is labelled with the name of the node and the name of the player who decides the move at that node; while the dots corresponding to the terminal nodes (outcomes) are labelled with the name of the node $\left(o_{1}, o_{2}, o_{3}, o_{4}\right)$ and with the players' payoffs, written as pairs $\left(p_{a}, p_{b}\right)$, where $p_{a}$ is Alice's payoff and $p_{b}$ is Bob's. Note that in this game there is one backward induction outcome, $o_{1}$, and furthermore that the unique backward induction strategy profile assigns to each $v_{m}$ the successor $o_{m+1}$.

\subsection{Language for games}

For any given game $G$, we define a set of basic (atomic) sentences $\Phi_{G}$ from which to build a language. First, we require $\Phi_{G}$ to contain a sentence for each leaf: for every $o \in \mathcal{O}$, there is a basic sentence $\bar{o}$. For simplicity, we often just write $o$, instead of $\bar{o}$. In addition $\Phi_{G}$ contains sentences to express the players' preferences over leaves: for each $i \in N$ and $\left\{o, o^{\prime}\right\} \subseteq \mathcal{O}, \Phi_{G}$ has a basic sentence $o \prec_{i} o^{\prime}$. Our formal language for games $G$ is simply the language APAL-CDL defined above, where the set of atomic sentences is the set $\Phi_{G}$. To talk about the non-terminal nodes, we introduce the following abbreviation:

$$
\bar{v}:=\bigvee_{v \rightsquigarrow o} o
$$

for any $v \in G-\mathcal{O}$. As for terminal nodes, we will often denote this sentence by $v$ for simplicity, instead of $\bar{v}$.

\subsection{Plausibility models for games}

We now turn to defining models for games. A plausibility model for game $G$ is just a plausibility model $\left(S, \leq_{i}, V\right)_{i \in N}$ for the set $\Phi_{G}$. We interpret every state $s \in S$ as an initial state in a possible play of the game. Intuitively, the sentence $\bar{o}$ is true at a state $s$ if outcome o will be reached during the play that starts at $s$; and the sentence $o \prec_{i} o^{\prime}$ says that player i 's payoff at $o$ is strictly smaller than her payoff at $o^{\prime}$.

Observe that nothing in our definition of models for $G$ guarantees that states come with a unique outcome or that the players know the set of outcomes! To ensure this (and 
Fig. 2 A game model $\mathcal{M}_{1}$ for the centipede game $G$

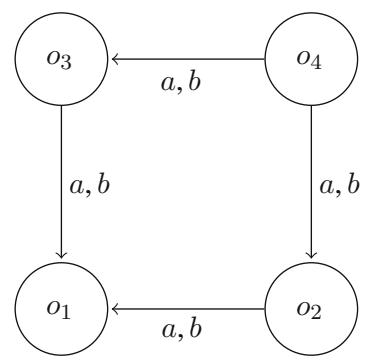

Fig. 3 A plausibility model $\mathcal{M}_{2}$ for $G$ which is not a "game model"

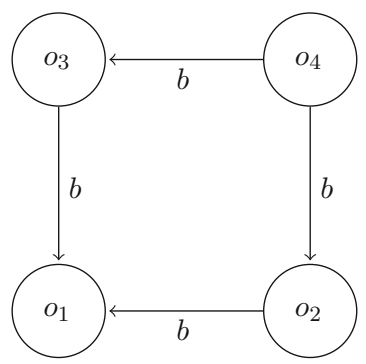

other desirable constraints), we later focus on a special class of plausibility models for a game, called "game models".

Examples Figures 2 and 3 represent two different plausibility models $\mathcal{M}_{1}$ and $\mathcal{M}_{2}$ for the centipede game $G$. Here, we use labelled arrows for the converse plausibility relations $\geq_{a}$ (going from less plausible to more plausible states), but for convenience we skip all the loops and all the arrows that can be obtained by transitivity.

Note that in the model $\mathcal{M}_{2}$, Alice (player a) knows the state of the world: in each state, she knows both the outcome and Bob's beliefs (and belief revision policy), i.e. the sentence $\bigwedge_{o \in \mathcal{O}}\left(\bar{o} \Rightarrow K_{a} \bar{o}\right)$ holds at all states of $\mathcal{M}_{2}$. But this is not true in model $\mathcal{M}_{1}$ : on the contrary, in $\mathcal{M}_{1}$ (it is common knowledge that) nobody knows the outcome of the game, and moreover nobody can exclude any outcome. Intuitively, the future is "epistemically open" in $\mathcal{M}_{1}$, but not in $\mathcal{M}_{2}$. However, we can also intuitively see that, in both models, (it is common knowledge that) all the players know the (structure of the) game: the available outcomes, the structure of the tree, the payoffs etc.

We now want to formalise our intuitions about open future and about having common knowledge of the structure of the game. To do this, we will focus on a special class of models, that we call "game models". Intuitively, each state of a game model comes with a complete play of the game, and hence it should have a uniquely determined outcome, and the set of possible outcomes as well as the players' preferences over them should be common knowledge. However, the players in this (initial) state should not have non-trivial knowledge about the outcome of the play. Indeed, they should have "freedom of choice" during the play, which means they can in principle play any move, so that at the outset of the play they cannot exclude a priori any outcomes. 


\subsection{Game models}

The class of game models for $G$, denoted by $\mathfrak{M}_{G}$, is the class of all plausibility model for $G$ satisfying the following conditions (for all players $i \in N$ ):

1. $\forall s \in S \exists ! o \in \mathcal{O}: s \in V(o)$

2. $V\left(o \prec_{i} o^{\prime}\right)= \begin{cases}S & \text { if } h_{i}(o)<h_{i}\left(o^{\prime}\right) \\ \emptyset & \text { otherwise }\end{cases}$

3. $\forall s \in S \forall o \in \mathcal{O}: V(o) \cap[s]_{i} \neq \emptyset$

The first condition entails that there is common knowledge of the set of possible outcomes, as well as of the fact that to each state is associated a unique actual outcome. This reflects the fact that the future, for each particular play (state), is determined. The second condition entails that the preferences over outcomes are commonly known. Finally, the third condition says that (it is common knowledge that) the future is epistemically open: in the initial state of any play, no player has "knowledge" (in the strong sense of "irrevocable", absolutely unrevisable knowledge) that any outcome is impossible. This is meant to apply even to the states that are incompatible with that player's plan of action.

\subsection{Open future}

We take condition (3) to embody the players' freedom of choice, as well as the possibility of error: in principle, players might always change their minds or make mistakes, hence any belief excluding some of the outcomes may have to be revised later. Even if we would assume (as is usually assumed) that players (irrevocably) know their own strategy, i.e. even if they are not allowed to change their minds, and even if we assume (as postulated by Aumann) that they have common knowledge of "rationality" (and so that they can exclude some obviously irrational choices), it still would not follow that they can completely exclude any outcome: mistakes can always happen, or players may always lose their rationality and become temporarily insane; so a rational plan does not necessarily imply a rational play, and hence the future still remains open.

Condition (3) is natural given our interpretation of the "knowledge" operator $K$ as representing hard information, that is absolutely certain and irrevocable. If any node is "known" (in this sense) to be unreachable, then that node should simply be deleted from the game tree: this just corresponds to playing a different game. So if a player $i$ were to irrevocably know that a given node is unreachable, then the structure of the game would not really be common knowledge: $i$ would in fact know that she is playing another game than $G$. Thus, one can consider the "open future" postulate as a natural strengthening of the "common knowledge of the game" assumption.

A different way to proceed would be to impose the above conditions only locally, at the "real" (initial) state of the play. Let Struct $_{G}$ be the following sentence, describing the "structure of the game" $G$ :

$$
\bigvee_{o \in \mathcal{O}} o \wedge \bigwedge_{o \neq o^{\prime} \in \mathcal{O}} \neg\left(o \wedge o^{\prime}\right) \wedge \bigwedge_{\begin{array}{c}
i \in N, o, o^{\prime} \in \mathcal{O} \\
\text { s.t. } h_{i}(o)<h_{i}\left(o^{\prime}\right)
\end{array}} o \prec_{i} o^{\prime} \wedge \bigwedge_{\begin{array}{c}
i \in N, o, o^{\prime} \in \mathcal{O} \\
\text { s.t. } h_{i}(o) \geq h_{i}\left(o^{\prime}\right)
\end{array}} \neg o \prec_{i} o^{\prime}
$$


Fig. 4 A game model $\mathcal{M}_{3}$ in which players don't have strategies

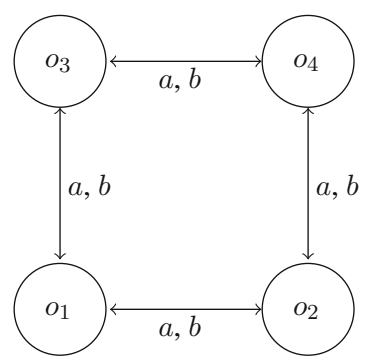

Similarly, let $\mathrm{F}_{G}:=\bigwedge_{o \in \mathcal{O}, i \in N} \neg K_{i} \neg o$ be the sentence saying that at the outset of game $G$ the future is epistemically open. Then our proposed "local" requirement is that in the initial state $s$ we have "common knowledge of the structure of the game and of open future", i.e. $s$ satisfies the sentence $C k\left(\operatorname{Struct}_{G} \wedge \mathrm{F}_{G}\right)$. Then it is easy to see that this "local" requirement is equivalent to the above global requirement of having a "game model": for every state $s$ in any plausibility model $\mathcal{M}$ for $G, s$ satisfies $C k\left(\right.$ Struct $\left._{G} \wedge \mathrm{F}_{G}\right)$ iff it is bisimilar ${ }^{6}$ to a state in some game model $\mathcal{M}^{\prime} \in \mathfrak{M}_{\mathcal{G}}$.

Examples Note that the model $\mathcal{M}_{1}$ from Fig. 2 is a game model, while $\mathcal{M}_{2}$ from Fig. 3 is not: indeed, in $\mathcal{M}_{2}$ it is common knowledge that Alice always knows the outcome, which contradicts the "Open future" assumption.

\subsection{Encoding strategies as conditional beliefs}

If a player adopts a particular (pure) strategy, our language can encode this in terms of the player's conditional beliefs about what she would do at each of her decision nodes. For instance, we say that Alice "adopts the backward induction strategy" in a given state $s$ of a model for the Centipede Game in Fig. 1 iff the sentences $B_{a} o_{1}$ and $B_{a}^{v_{2}} o_{3}$ hold at state $s$. Similarly, we can express the fact that Bob adopts a particular strategy, and by putting these together we can capture strategy profiles. A given profile is realized in a model if the correspondent sentence is true at a state of that model.

Note that, in our setting, nothing forces the players to adopt (pure) strategies. Strategies are "complete" plans of action prescribing a unique choice (a belief that a particular move will be played) for each decision node of the player. But the players might simply consider all their options as equi-plausible, which essentially means that they do not have a strategy.

Examples In (any state of) model $\mathcal{M}_{1}$ from Fig. 2, it is common knowledge that both players adopt their backward induction strategies. In contrast, in the model $\mathcal{M}_{3}$ from Fig. 4, it is common knowledge that no player has a strategy (at any node):

So the assumption that players have (pure) "strategies" is an extremely strong assumption, which we will not need. There is no a priori reason to assume (and there are good empirical reasons to reject) that players play according to fully-determined strategies. Our models are general enough to dispense with this assumption; indeed,

\footnotetext{
${ }^{6}$ Here, "bisimilarity" is the standard notion used in modal logic, applied to plausibility models viewed as Kripke models with atomic sentences in $\Phi$ and with relations $\leq_{i}$. The important point is that our language APAL-CDL cannot distinguish between bisimilar models and states.
} 
our work shows that this assumption is not needed for proving (common belief) that the backward induction strategy is played.

\subsection{Intentions as beliefs}

In the above discussion, we identified an agent's intentions with her beliefs about what she is going to do, and so we represented the decision maker's plan of action as a belief about her (future) action. This identification is philosophically debatable, since agents may be aware of the possibility of mistakes, and so they may doubt that their intentions will be realized. But one can also argue that, in the context of Game Theory, such distinctions will be of very limited significance: indeed, an intention that is not believed to be enforceable is irrelevant for strategic planning (though see Roy 2008 for a discussion of intentions in game theory). The players only need to know each others' beliefs about their future actions and about each others' beliefs etc., in order to make their own rational plans; whether or not they are being informed about each others' (completely unenforceable and not believed to be enforceable) "intentions" will not make any difference. So, for the purposes of this paper, we can safely adopt the simplifying assumption that the agents believe that they will be able to carry out their plans. Given this assumption, an agent's "intentions" can be captured by her beliefs about her (future) actions.

\subsection{Representing players' evolving beliefs}

Recall that we think of every state of a game model $\mathcal{M}_{G} \in \mathfrak{M}_{G}$ as an initial state (of a possible play) of the game $G$. As the play goes on, the players' hard and soft information, their knowledge and beliefs, evolve. To represent this evolution, we will need to successively change our model, so that e.g. when a node $v$ is reached, we want to obtain a corresponding model of the subgame $G^{v}$. That is precisely, in this perfect information setting, what is achieved by updating the model with public announcements: indeed, in a game of perfect information, every move, say from a node $u$ to one of its immediate successors $u^{\prime}$, can be "simulated" by a public announcement $! u^{\prime}$. In this way, for each subgame $G^{v}$ of the original model $\mathcal{M}$, we obtain a model $\mathcal{M}^{v}$, that correctly describes the players' knowledge and beliefs at the moment when node $v$ is reached during a play. This is indeed a model of the corresponding subgame $G^{v}$ :

Proposition 2.1 If $\mathcal{M} \in \mathfrak{M}_{G}$ then $\mathcal{M}^{v} \in \mathfrak{M}_{G^{v}}$.

Example Consider a play of the Centipede game $G$ that starts in the initial situation described by the model $\mathcal{M}_{1}$ in Fig. 2 , and in which the real state of the world is the one having outcome $o_{2}$ : so Alice first plays "right", reaching node $v_{1}$, and the Bob plays "down", reaching the outcome $o_{2}$. The model $\mathcal{M}_{1}$ from Fig. 2 gives us the initial situation, the model $\mathcal{M}_{1}^{v_{1}}$ in Fig. 5 describes the epistemic situation after the first move, and then the model $\mathcal{M}_{1}^{o 2}$ in Fig. 6 gives the epistemic situation at the end of the play:

In this way, for each given initial state $s$ (of a given play $v_{0}, v_{1}, \ldots, o$ of the game, where $o$ is the unique outcome such that $s \in V(o)$ ), we obtain a sequence of evolving 
Fig. 5 The model $\mathcal{M}_{1}^{v_{1}}$

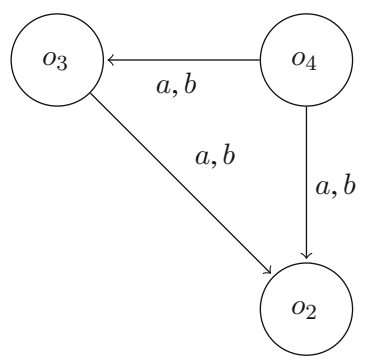

Fig. 6 The model $\mathcal{M}_{1}^{o_{2}}$

game models

$$
\mathcal{M}=\mathcal{M}^{v_{0}}, \mathcal{M}^{v_{1}}, \ldots, \mathcal{M}^{o}
$$

describing the evolving knowledge and beliefs of the players during any play. Each model $\mathcal{M}^{v}$ accurately captures the players' beliefs at the moment when node $v$ is reached. Note also that every such sequence ends with a model $\mathcal{M}^{o}$ consisting of only one node (a leaf $o$ ); this reflects the fact that at the end of the game, there is no uncertainty left: the outcome, as well as the whole history of the game, are now common knowledge.

\subsection{Simulating moves by public announcements}

Using the dynamic "public announcement" modalities in constructs such as $[! v] B_{i}$, we can talk, at the initial state $s \in \mathcal{M}$ and without leaving the original model $\mathcal{M} \in \mathfrak{M}_{G}$, about all these future, evolving beliefs of the players at nodes $v$ other than the initial node $v_{0}$. Indeed, in a game of perfect information, all the moves are public. So the epistemic effect of a move to node $v$ is the same as that of a truthful public announcement $! v$ (saying that the node $v$ is reached during the play). In other words, we can "simulate" moves in games of perfect information by truthful public announcements. ${ }^{7}$

\section{Dynamic rationality in decisions and games}

We now define our fundamental notions of dynamic rationality and rational play. First we will look at single-agent (one-step) decision situations, and then at interactive decision situations, i.e. games.

\footnotetext{
${ }^{7}$ We believe that the more general case, of games of imperfect information, can also be handled by using other kinds of epistemic actions proposed in Dynamic Epistemic Logic (Baltag et al. 1999). But we leave this development for future work.
} 


\subsection{Single agent decision problems}

Given a one-step decision problem $\mathcal{P}$ with a set of outcomes $\mathcal{O}$, the decision-maker $i$ selects one of the outcomes $o \in \mathcal{O}$. The decision-maker may have various hard and soft information about which outcomes can actually be realized and which not. This will determine her knowledge and her beliefs. We assume that her "hard" knowledge restricts her possible choices: she can only select outcomes that she doesn't know to be impossible.

What this amounts to is the following: for the decision maker $i$, the "true" set of possible outcomes is $\left\{o \in \mathcal{O} \mid \neg K_{i} \neg o\right\}$, i.e. the set of all the "epistemically possible" outcomes. So her selected option must satisfy: $o \in\left\{o \in \mathcal{O} \mid \neg K_{i} \neg o\right\}$. This allows us to capture the "selection" problem using epistemic operators.

To assess whether the decision is "rational" or not, one considers the decisionmaker's subjective preferences, modelled as a total pre-order $\preccurlyeq_{i}$ on $\mathcal{O}$. We assume that agents know their preferences; indeed, these are interpreted as "doxastic" preferences: beliefs about what's best. Given this interpretation, the CDL postulation of Full Introspection (of beliefs) implies that agents know their preferences.

\subsubsection{Rational choice}

Rationality, in this case, corresponds to requiring that the selected option is not worse than any other (epistemically) possible alternative. In other words, $i$ 's solution of the decision problem $\mathcal{P}$ is rational if she does not choose any option that is strictly less preferable than an option she doesn't know to be impossible:

$$
\mathrm{R}_{i}^{\mathcal{P}}:=\bigwedge_{o, o^{\prime} \in \mathcal{O}}\left(o \prec_{i} o^{\prime} \wedge \neg K_{i} \neg o^{\prime} \Rightarrow \neg o\right)
$$

The main difference between our definition and the standard definition of rational decision-making is the epistemic limitation of the choice set. The epistemic operators are used here to delimit what is currently known about the availability of options: $i$ 's choice should only be compared against options that are not known to be unavailable. This is an important difference, and its importance becomes clear when we generalise our definition to extensive games, cf. the difference between 'dynamic' rationality and traditional 'substantive' rationality, described below.

\subsection{Extensive games}

We now aim to extend the above definitions to the case of multi-agent many-stage decisions, i.e. extensive games (of perfect information). Recall that in an extensive game we are given the players' subjective preferences $\preccurlyeq_{i}$ only over the leaves. However, at all the intermediate stages of the game, players have to make local choices, not between "final" outcomes, but between "intermediary" outcomes, that is: between other nodes of the game tree. 
So, in order to assess players' rationality, we need to extend the subjective preference relations to all the nodes of the game tree. Fortunately, given the above doxastic interpretation of preferences, there is an obvious (and natural) way to define these extensions. Namely, a player considers a node $u$ to be strictly less preferable to a node $u^{\prime}$ if she believes the first to be strictly dominated by the second. More precisely, if every outcome that she believes to be achievable given that $u$ is reached is worse than every outcome that she believes to be achievable given that $u^{\prime}$ is reached:

$$
u \prec_{i} u^{\prime}:=\bigwedge_{o, o^{\prime} \in \mathcal{O}}\left(\neg B_{i}^{u} \neg o \wedge \neg B_{i}^{u^{\prime}} \neg o^{\prime} \Rightarrow o \prec_{i} o^{\prime}\right)
$$

By the Full Introspection of beliefs (a postulate of the logic $C D L$ ), it follows that we still have that players know their extended preferences over all the nodes of the game.

\subsubsection{Rationality at a node}

Each node $v \in G_{i}$ can be considered as a (distinct) decision problem, in which the decision-maker is $i$, the set of outcomes is the set $\{u \in G: v \rightarrow u\}$ of all immediate successors of $v$, and the subjective preference relation is given by the (restriction of the) extended relation $\prec_{i}$ defined above (to the set $\{u \in G: v \rightarrow u\}$ ). So we can define the rationality of a player $i$ at a node $v \in G_{i}$ as rationality for the corresponding decision problem, i.e. the player's selection at each decision node consists only of "best answers". Note that, as before, the player's choice is epistemically limited: if she has "hard knowledge" excluding some successors (for instance, because those nodes have already been bypassed), then those successors are excluded from the set of possible options. The only difference is that the "knowledge" involved is the one the agent would have at that decision node, i.e. it is conditional on that node being reached. Formally, we obtain:

$$
\mathrm{R}_{i}^{v}:=\bigwedge_{u, u^{\prime} \leftarrow v}\left(u \prec_{i} u^{\prime} \wedge \neg K_{i}^{v} \neg u^{\prime} \Rightarrow \neg u\right)
$$

where $K_{i}^{\varphi} \psi:=K_{i}(\varphi \Rightarrow \psi)$.

\subsubsection{Dynamic rationality}

Let $\mathrm{R}_{i}$ be the sentence

$$
\mathrm{R}_{i}=\bigwedge_{v \in G_{i}} \mathrm{R}_{i}^{v}
$$

If $\mathrm{R}_{i}$ is true, we say that player $i$ satisfies dynamic rationality. By unfolding the definition, we see it is equivalent to:

$$
\mathrm{R}_{i}=\bigwedge_{v \in G_{i}} \bigwedge_{u, u^{\prime} \leftarrow_{i} v}\left(u \prec_{i} u^{\prime} \wedge \neg K_{i}^{v} \neg u^{\prime} \Rightarrow \neg u\right) .
$$


As we'll see, asserting this sentence at a given moment is a way of saying that the player will play rationally from that moment onwards, i.e. she will make the best move at any current or future decision node.

In the following, "Dynamic Rationality" denotes the sentence

$$
\mathrm{R}:=\bigwedge_{i} \mathrm{R}_{i}
$$

saying that all players are dynamically rational.

\subsubsection{Comparison with substantive rationality}

To compare our notion with Aumann's concept of "substantive rationality", we have to first adapt Aumann's definition to a belief-revision context. This has already been done by a number of authors e.g. Battigalli and Siniscalchi (1999, 2002), resulting in a definition of "rationality at a node" that differs from ours only by the absence of epistemic qualifications to the set of available options (i.e. the absence of the term $\neg K_{i}^{v} \neg u^{\prime}$ ). The notion of substantive rationality is then obtained from this in the same way as dynamic rationality, by quantifying over all nodes, and it is thus equivalent to the following definition:

$$
\mathrm{SR}_{i}=\bigwedge_{v \in G_{i}} \bigwedge_{u, u^{\prime} \leftarrow_{i} v}\left(u \prec_{i} u^{\prime} \Rightarrow \neg u\right)
$$

It is obvious that substantive rationality implies dynamic rationality

$$
\mathrm{SR}_{i} \Rightarrow \mathrm{R}_{i}
$$

but the converse is in general false. To better see the difference between $\mathrm{SR}_{i}$ and $\mathrm{R}_{i}$, recall that a formula being true in a model $\mathcal{M} \in \mathfrak{M}_{G}$ means that it is true at the first node (the root) of the game tree $G$. However, we will later have to evaluate the formulas $\mathrm{R}_{i}$ and $\mathrm{SR}_{i}$ at other nodes $w$, i.e. in other models of the form $M^{w}$ (models for subgames $G^{w}$ ). Since the players' knowledge and beliefs evolve during the game, what is (not) known/believed conditional on $v$ in model $M^{w}$ differs from what was (not) known/believed conditional on $v$ in the original model (i.e. at the outset of the game). In other words, the meaning of both dynamic rationality $\mathrm{R}_{i}$ and substantive rationality $\mathrm{SR}_{i}$ will change during a play. But they change in different ways. At the initial node $v_{0}$, the two notions are equivalent. But, once a node $v$ has been bypassed, or once the move at $v$ has already been played by a player $i$, that player is counted as rational at node $v$ according to our definition, while according to the usual (non-epistemically qualified) definition the player may have been irrational at $v$.

In other words, the epistemic limitations we imposed on our concept of dynamic rationality make it into a future-oriented concept. At any given moment, the rationality of a player depends only on her current beliefs and knowledge, and so only on the options that she currently considers possible: past, or by-passed, options are irrelevant. Dynamic Rationality simply expresses the fact that the player's decision 
in any future contingencies is rational (given her future options and beliefs). Unlike substantive rationality, our concept has nothing to do with the past or with contingencies that are known to be impossible: a player $i$ may still be "rational" in our sense at a given moment/node $v$ even when $v$ could only have been reached if $i$ has already made some "irrational" move. The (knowledge of some) past mistake(s) may of course affect the others' beliefs about this player's rationality; but it doesn't directly affect her rationality, and in particular it doesn't automatically render her irrational.

\subsubsection{Solving the BI paradox}

As explained above, our concept is very different from (and, arguably, more realistic than) Aumann's and Stalnaker's substantive rationality, but also from other similar concepts in the literature (for example Rabinowicz (1998) "habitual" or "resilient" rationality, etc). The difference becomes more apparent if we consider the assumption that "rationality" is common belief, in the strongest possible sense, including common "strong" belief (in the sense of Battigalli and Siniscalchi 2002), common persistent belief, or even common "knowledge" in the sense of Aumann. As correctly argued by Stalnaker and Reny, these assumptions, if applied to the usual notions of rationality in the literature, bear no relevance for what the players would do (or believe) at the nodes that are incompatible with these assumptions! The reason is that, if these counterfactual nodes were to be reached, then by that time the belief in "rationality" would have already been publicly disproved: we cannot even entertain the possibilities reachable by irrational moves except by suspending our belief (or "knowledge") in rationality. Hence, the above assumptions cannot tell us anything about the players' behaviour or rationality at such counterfactual nodes, and thus they cannot be used to argue for the plausibility of the backward induction solution (even if they logically imply it)! In contrast, our notion of dynamic rationality is not automatically disproved when we reach a node excluded by common belief in it: a player may still be rational with respect to her current and future options and decisions even after making an "irrational" move. Indeed, the player may have been playing irrationally in the past, or may have had a moment of temporary irrationality, or may have made some mistakes in carrying out her rational plan; but she may have recovered now and may play rationally thereafter. Since our notion of rationality is future-oriented, no information about past moves will necessarily and automatically shatter belief in rationality (although of course it may still shatter it, or at least weaken it). So it is perfectly consistent (although maybe not always realistic) to assume that players maintain their common belief in dynamic rationality despite all past failures of rationality. In fact, this is our proposed solution to the BI paradox: we will show that such a "stable" common belief in dynamic rationality (or more precisely, common knowledge of the stability of the players' common belief in rationality) is exactly what is needed to ensure common belief in the backward induction outcome!

\subsubsection{Rational planning}

A weaker condition requires only that, for each decision node $v$, the option that the decision-maker is planning at $v$ to select (at $v$ ) is the best, given the other (epistemi- 
cally) possible alternatives. By identifying as above the players' plans of actions with their beliefs about their actions, we can thus say that a decision maker is a rational planner in the game $G$ if at each decision node she believes that she will take "the best decision”, even if in the end she may accidentally make a wrong choice:

$$
\mathrm{RP}_{i}:=\bigwedge_{v \in G_{i}} B_{i}^{v} \mathrm{R}_{i}^{v}
$$

By unfolding the definition, we see it is equivalent to:

$$
\mathrm{RP}_{i}=\bigwedge_{v \in G_{i}} \bigwedge_{u, u^{\prime} \leftarrow{ }_{i} v}\left(u \prec_{i} u^{\prime} \wedge \neg K_{i}^{v} \neg u^{\prime} \Rightarrow B_{i}^{v} \neg u\right)
$$

\subsubsection{No mistakes}

$\mathrm{RP}_{i}$ only states that the decision maker $i$ has a rational plan for current and future contingencies. But mistakes can happen, so if we want to ensure that the decision that is actually taken is rational we need to require the player makes no mistakes in carrying out her plan:

$$
\text { No-Mistakes }_{i}:=\bigwedge_{v \in G_{i}} \bigwedge_{u \leftarrow v}\left(B_{i}^{v} \neg u \Rightarrow \neg u\right)
$$

The sentence No-Mistakes $i$ says that player $i$ 's decision are always consistent with her "plan": she never plays a move that, at the moment of playing, she believed won't be played.

As expected, the conjunction of "rational planning" and "no mistakes" entails "rational playing" (i.e., "dynamic rationality"):

$$
\mathrm{RP}_{i} \wedge \text { No-Mistakes }_{i} \Rightarrow \mathrm{R}_{i}
$$

\section{Backward induction in games of perfect information}

It is easy to see that Aumann's theorem stating that common knowledge of substantive rationality implies the backward induction outcome (Aumann 1995) can be strengthened to the following

Proposition 4.1 In any state of any plausibility model for a game of perfect information, common knowledge of dynamic rationality implies the backward induction outcome.

Unfortunately, common knowledge of (either dynamic or substantive) rationality can never hold in a game model: it is simply incompatible with the "EpistemicallyOpen Future" condition. By requiring that players have "hard" information about the outcome of the game, Aumann's assumption does not allow them to reason hypothetically or counterfactually about other possible outcomes, at least not in a consistent 
manner. ${ }^{8}$ This undermines the intuitive rationale behind the backward induction solution, and it is thus open to Stalnaker's criticism.

So in this section, we are looking for natural conditions that can be satisfied on game models, but that still imply the backward induction outcome (or at least common belief in it). One such condition is common knowledge of (general) stable belief in (dynamic) rationality: $C k[$ ! $] E b R$. This is in fact a "strong" form of common belief, being equivalent to $C k[$ ! $] C b R$, i.e. to common knowledge of stable common belief in rationality.

Theorem 4.2 The following holds in any state $s$ of any game model $\mathcal{M} \in \mathfrak{M}_{G}$ :

$$
C k[!] E b \mathrm{R} \Rightarrow C b\left(B I_{G}\right)
$$

where $B I_{G}:=\bigvee\left\{o \mid o \in B I_{G}\right\}$ is the sentence saying that the current state determines a backward-induction outcome in the game G. Equivalently, the following formula is valid over plausibility frames for the game $G$ :

$$
C k\left(\operatorname{Struct}_{G} \wedge F_{G} \wedge[!] C b \mathrm{R}\right) \Rightarrow C b\left(B I_{G}\right)
$$

In English: assuming common knowledge of the game structure and of open future, if it is common knowledge that, no matter what new (truthful) information the players may (jointly) learn during the game (i.e. no matter what is played), general belief in rationality will be maintained, then it is common belief that the backward induction outcome will be reached. If we define "stable common belief" in a proposition $P$ as $[!] C b P$, then we can give a more concise English formulation of the above theorem: common knowledge of the game structure, of open future and of stable common belief in dynamic rationality implies common belief in the backward-induction outcome.

Although rationality cannot be common knowledge in a game model, rational planning can be. When this is the case, we obtain the following

Corollary 4.3 In a game model, common knowledge of "rational planning" and of stable belief in "no mistakes" implies the backward-induction outcome; i.e. the formula

$$
C k(\mathrm{RP} \wedge[!] E b \text { No-Mistakes }) \Rightarrow C b\left(B I_{G}\right)
$$

is valid on game models.

The above results only give us common belief in the backward-induction outcome, but nothing ensures that this belief is correct. If we want to ensure that the backwardinduction outcome is actually played, we need to add the requirement that the (stable common) belief in rational play assumed in the premise is correct, i.e. that players actually play rationally:

\footnotetext{
${ }^{8}$ Indeed, if $o$ is the backward induction outcome, then the above Proposition entails $K_{i} o$ for all players $i$, and thus for every other outcome $o^{\prime} \neq o$ and every proposition $P$, we have $B_{i}^{o^{\prime}} P$ : the players believe everything (including inconsistencies) conditional on $o^{\prime}$.
} 
Theorem 4.4 The following holds in any state s of any game model $\mathcal{M} \in \mathfrak{M}_{G}$ :

$$
\mathrm{R} \wedge C k[!] E b \mathrm{R} \Rightarrow B I_{G}
$$

\subsection{No strategies!}

Observe that we did not assume that the players have complete (pure) "strategies". That is, we do not insist that they have fully determined plans of action, uniquely specifying one move for at each decision node, but only that they have partial plans, i.e. incomplete beliefs about what moves they should play: at each decision node they choose a set of moves rather than one unique move. So an important side-result of our work is that the assumption that players have (complete, pure) strategies is not necessary for proving backward-induction results.

\subsection{Ensuring backward-induction strategy profile}

If, however, we want to postulate that every player does have a (complete, pure) strategy, we need to say that, for each node $v$ of her choice, there exists a (unique) immediate successor $u$ that she believes will be played if $v$ is reached (i.e. she plans to play $u$ at $v$ ):

$$
\text { Strategies }:=\bigwedge_{i} \bigwedge_{v \in G_{i}} \bigvee_{u \leftarrow i} B_{i}^{v} u \text {. }
$$

In cases where Strategies is common knowledge as well, we can strengthen the Theorem 4.2 to:

Corollary 4.5 The following holds in any state s of any game model $\mathcal{M} \in \mathfrak{M}_{G}$ :

$$
C k(\text { Strategies } \wedge[!] E b \mathrm{R}) \Rightarrow C b\left(\text { BI-Profile }_{G}\right)
$$

where BI-Profile ${ }_{G}$ is the sentence saying that the strategies given by each player's conditional beliefs in the initial state s form a backward-induction profile.

Finally, the following theorem ensures that above results are not vacuous:

Theorem 4.6 For every extensive game $G$, there is a game model $\mathcal{M} \in \mathfrak{M}_{G}$ and a state $s \in \mathcal{M}$ satisfying the sentence

$$
\text { No-Mistakes } \wedge C k(\mathrm{RP} \wedge \text { Strategies } \wedge[\text { ! }] \text { EbNo-Mistakes }) \text {. }
$$

As a consequence, the sentence $\mathrm{R} \wedge C k[!] E b \mathrm{R} \wedge C k$ Strategies is also satisfied.

The proofs of these theorems are in the next section. Some alternative (weaker) conditions ensuring the backward induction outcome are given in Sect. 6 . 


\section{Proofs}

Definition 5.1 For a finite set $\mathcal{O}$ of "outcomes" and a finite set $P$ of "players", we denote by $\mathfrak{G} \mathfrak{a m e s}(\mathcal{O}, P)$ the class of all perfect information games having any subset of $\mathcal{O}$ as their set of outcomes and having any subset of $P$ as their set of players.

Definition 5.2 For any sentence $\varphi$ of our language,

$\varphi$ is valid on a game $G$ if $\varphi$ is true at every state $s$ of every game model $\mathcal{M} \in \mathfrak{M}_{G}$. $\varphi$ is valid over $\mathfrak{G a m e s}(\mathcal{O}, P)$ if $\varphi$ is valid on every game $G \in \mathfrak{G} \mathfrak{a m e s}(\mathcal{O}, P)$.

When the game $G$ is implicit from the context, we will often abbreviate $B I_{G^{u}}$, i.e. the name for the formula that defines all the backward-induction outcomes in the subgame of $G$ that starts at the node $u$, to $B I^{u}$.

Lemma 5.3 For every perfect information game $G$, if we denote the root of $G$ by $v_{0}$, the first player of $G$ (playing at $v_{0}$ ) by $i$ and the first move of $i$ (the successor node played at $v_{0}$ ) by $v_{1}$, then the sentence

$$
\mathrm{R}_{i}^{v_{0}} \wedge \bigwedge_{u \leftarrow v_{0}} B_{i}^{u}[! u] B I^{u} \wedge\left[! v_{1}\right] B I^{v_{1}} \Rightarrow B I
$$

is valid on $G$.

Proof This follows directly from the definition of rationality at a node and the definition of $B I$. The assumption that $B_{i}^{u}[! u] B I^{u}$ is true at $s$ means that all the states (deemed as "most plausible by $i$ conditional on $u$ ") in the set $s_{i}^{u}:=\min _{\leq i}\left(\bar{u} \cap[s]_{i}\right)$ have only outcomes that are backward induction outcomes in the corresponding subgame: i.e. we have $o(t) \in B I_{G^{u}}$ for all $t \in s_{i}^{u}$. Given that all these outcomes $\left\{u: u \leftarrow v_{0}\right\}$ are consistent with $i$ 's knowledge (since we are in a game model), the fact that $i$ is rational at $v_{0}$ implies that the successor node $v_{1}$ chosen by $i$ must be one that maximises her payoff $h_{i}\left(o\left(s_{i}^{u}\right)\right)$ among all the outcomes in $\bigcup_{u \leftarrow v_{0}} B I_{G^{u}}$. But, by the definition, such a node $v_{1}$ is exactly the choice prescribed at $v_{0}$ by the backward induction strategy! Given this backward-induction choice $\left(v_{1}\right)$ of $i$ at node $v_{0}$, and given the fact (ensured by the condition $\left[! v_{1}\right] B I^{v_{1}}$ ) that starting from node $v_{1}$ everybody will play the backward induction choices, we can conclude that the outcome $o(s)$ belongs to the backward induction set of outcomes $B I_{G^{v_{0}}}=B I_{G}$ for the game $G$. Hence $s$ satisfies $B I_{G}$.

The Main Lemma underlying our results is the following:

Lemma 5.4 ("Main Lemma") Fix a finite set $\mathcal{O}$ of outcomes and a finite set $P$ of players. Let $\varphi$ be any sentence in our language APAL-CDL having the following property: for every game $G \in \mathfrak{G} \mathfrak{a m e s}(\mathcal{O}, P)$, if we denote the root of $G$ by $v_{0}$, the first player of $G$ (playing at $v_{0}$ ) by $i$ and the first move of $i$ (the successor node played at $v_{0}$ ) by $v_{1}$, then the sentence

$$
\varphi \Rightarrow R_{i}^{v_{0}} \wedge \bigwedge_{u \leftarrow v_{0}} B_{i}^{u}[! u] \varphi \wedge\left[! v_{1}\right] \varphi
$$


is valid on G. Given this condition, we have that the sentence

$$
\varphi \Rightarrow B I_{G}
$$

is valid over $\mathfrak{G} \mathfrak{a m e s}(\mathcal{O}, P)$.

Proof We need to prove that, for every game $G \in \mathfrak{G} \mathfrak{a m e s}(\mathcal{O}, P)$, the sentence $\varphi \Rightarrow$ $B I_{G}$ is valid on $G$. The proof is by induction on the length of the game $G$.

For games of length 0 (only one outcome, no available moves), the claim is trivial (since the only possible outcome is by definition the backward induction outcome).

Let $G$ be now a game of length $n>0$, and assume the claim is true for all games of smaller length. Let $v_{0}$ be the root of $G, i$ be the first player of $G, \mathcal{M} \in \mathfrak{M}_{G}$ be a game model for $G$ and $s$ be a state in $\mathcal{M}$ such that $s=\mathcal{M} \varphi$.

Let $u$ be any arbitrary immediate successor of $v_{0}$ (i.e. any node such that $u \leftarrow v_{0}$ ). By the property assumed in the statement of this Lemma, we have that $s \models \mathcal{M} B_{i}^{u}[! u] \varphi$, and so (if $s_{i}^{u}$ is the set defined in the proof of the previous Lemma, then) we have $t \models \mathcal{M}[! u] \varphi$ for all $t \in s_{i}^{u}$. Hence, we have $t \models \mathcal{M}^{u} \varphi$ for all $t \in s_{i}^{u} \cap \bar{u}$. By the induction hypothesis, we must have $t \models \mathcal{M}^{u} B I^{u}$ (since $\mathcal{M}^{u}$ is a game model for $G^{u}$, which has length smaller than $G$, and so the implication $\varphi \Rightarrow B I^{u}$ is valid on $\mathcal{M}^{u}$ ), for all $t \in s_{i}^{u} \cap \bar{u}$. From this we get that $t \models \mathcal{M}[! u] B I^{u}$ for all $t \in s_{i}^{u}$, and hence that $s \models \mathcal{M} B_{i}^{u}[! u] B I^{u}$.

Let $v_{1}$ be now the first move of the game in state $s$ (i.e. the unique immediate successor $v_{1} \leftarrow v_{0}$ such that $\left.s \models \mathcal{M} v_{1}\right)$. By the property assumed in this Lemma, we have that $s \models \mathcal{M}\left[! v_{1}\right] \varphi$. By the same argument as in the last paragraph, the induction hypothesis gives us that $s \models \mathcal{M}\left[! v_{1}\right] B I^{v_{1}}$. Putting together with the conclusion of the last paragraph and with the fact (following from the Lemma's hypothesis) that $\varphi \Rightarrow R_{i}^{v_{0}}$ is valid on $\mathcal{M}$, we infer that $s \models \mathcal{M} R_{i}^{v_{0}} \wedge \bigwedge_{u \leftarrow v_{0}} B_{i}^{u}[! u] B I^{u} \wedge\left[! v_{1}\right] B I^{v_{1}}$. The desired conclusion follows now from Lemma 5.3.

Lemma 5.5 The sentence

$$
\varphi:=\mathrm{R} \wedge C k[!] E b \mathrm{R}
$$

has the property assumed in the statement of Lemma 5.4.

Proof The claim obviously follows from the following three sub-claims:

1. dynamic rationality is a "stable" property, i.e. the implication $\mathrm{R} \Rightarrow \bigwedge_{u}[! u] \mathrm{R}$ is valid;

2. the implication $C k[!] E b \psi \Rightarrow B_{i}^{u}[! u] C k[!] E b \psi$ is valid, for all formulas $\psi$ and all nodes $u \in G$;

3. the implication $C k[!] E b \psi \Rightarrow[! u] C k[!] E b \psi$ is valid, for all formulas $\psi$ and all nodes $u$.

All these claims are easy exercises in dynamic-epistemic logic. The first follows directly from the definition of dynamic rationality.

The second sub-claim goes as follows: assume that we have $C k[!] E b \psi$ at some state of a given model; then we also have $C k[! u][!] E b \psi$ for any node $u$ (since [!] $\theta$ 
implies $[! u][!] \theta$ ), and so also $K_{i} C k[! u][!] E b \psi$ (since common knowledge implies knowledge of common knowledge), from which we get $B_{i}^{u} C k[! u][!] E b \psi$ (because knowledge implies conditional belief under any conditions). This is the same as $B_{i}^{u}(u \rightarrow C k[! u][!] E b \psi)$, which implies $B_{i}^{u}\left(u \rightarrow C k^{u}[! u][!] E b \psi\right)$ (since common knowledge implies conditional common knowledge). But this last clause is equivalent to $B_{i}^{u}[! u] C k[!] E b \psi$ (by the Reduction Law for common knowledge after public announcements).

The third sub-claim goes as follows: assume that we have $C k[!] E b \psi$ in some state of a given model; then as before we also have $C k[! u][!] E b \psi$, and thus $C k^{u}[! u][!] E b \psi$ (since common knowledge implies conditional common knowledge). From this we

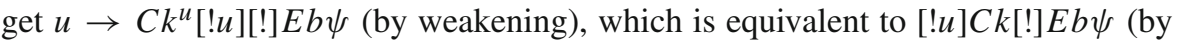
the Reduction Law for common knowledge after public announcements).

\section{Theorems 4.4 and 4.2}

Proof Theorem 4.4 follows now from Lemma 5.4 and Lemma 5.5. Theorem 4.2 follows from Theorem 4.4, by applying the operator $C k[$ !] $E b$ to both its premiss and its conclusion, and noting that the implication

$$
C k[!] E b \psi \Rightarrow C k[!] E b C k[!] E b \psi
$$

is valid.

\section{An alternative condition: common stable true belief in dynamic rationality}

The epistemic condition $\mathrm{R} \wedge C k[$ ! $] E b \mathrm{R}$ given above is not the weakest possible condition that ensures the backward induction outcome. Any property $\varphi$ satisfying the condition of our Main Lemma (Lemma 5.4) would do it. In particular, there exists a weakest such condition (the smallest event $E \subseteq S$ such that $E \subseteq R_{i}^{v_{0}} \cap \bigcap_{u \leftarrow v_{0}} B_{i}^{u}[! u] E \cap$ $\left.\left[! v_{1}\right] E\right)$, but it is a very complicated and unnatural condition. The one given above seems to be the simplest such condition expressible in our language APAL-CDL.

However, one can give weaker simple conditions if one is willing to go a bit beyond the language APAL-CDL, by adding fixed points for other (definable) epistemic operators.

Let stable true belief be a belief that is known to be a stable belief and it is also a stably true belief. Formally, we define:

$$
\operatorname{St}_{i} \varphi:=K_{i}[!] B_{i} \varphi \wedge[!] \varphi
$$

Thus stable true belief is stable belief in a stably true proposition. Stable true belief is a form of "knowledge", since it implies truth and belief:

$$
\operatorname{St}_{i} \varphi \Rightarrow \varphi \wedge B_{i} \varphi
$$

We can also think of stable true belief as an epistemic attitude towards the stability of a proposition: clearly it implies stably truth $\left(S_{t} b_{i} \varphi \Rightarrow[!] \varphi\right)$, but furthermore, knowledge 
that something is stably true implies stable true belief in it.

$$
K_{i}[!] \varphi \Rightarrow S t b_{i} \varphi
$$

Stable true belief is inherently a "positively introspective" attitude, i.e.

$$
S t b_{i} \varphi \Rightarrow S t b_{i} S t b_{i} \varphi
$$

but it is not positively introspective with respect to ("hard") knowledge:

$$
\operatorname{St}_{i} \varphi \not K_{i} S t b_{i} \varphi \text {. }
$$

Stable true belief is not negatively introspective, neither inherently nor with respect to knowledge.

If we restrict our attention to only ontic (i.e. non-doxastic) facts $p$, then we cannot detect the subtleties of stable true belief, and the difference between this concept, "stable belief" (simpliciter) and "safe belief". Notice in particular that, when applied to ontic facts $p$, stable true belief of $p$ is just the same as stable belief of $p$ and the same as the "safe belief" in $p$ from Baltag and Smets (2008b) which is the same as what Stalnaker calls "knowledge" (Stalnaker 2006). However, it is typical of interactive epistemology that one is not in general interested in epistemic/doxastic attitudes towards ontic facts, but in attitudes towards propositions that in turn depend on other attitudes. Examples of such higher-level attitudes are the important game-theoretic notions of "common knowledge of (or common belief in) rationality", "common knowledge of stable belief in rationality" and "common stable true belief in rationality": exactly the notions that interest us in this paper!

We can define common stable true belief in the same way as common knowledge: first define general stable true belief

$$
E \operatorname{stb} \varphi=\bigwedge_{i \in P} S t b_{i} \varphi
$$

(“everybody has stable true belief"), then put

$$
C s t b \varphi=\bigwedge_{n}(E s t b)^{n} \varphi
$$

Note that this definition, although semantically meaningful, is not a definition in our language APAL-CDL, since it uses infinite conjunctions. Indeed, we conjecture that common stable true belief is undefinable in the language APAL-CDL, since it doesn't seem to be expressible as a combination of common knowledge, common belief and dynamic operators.

Lemma 6.1 The sentence CstbR satisfies the condition of our Main Lemma (Lemma 5.4).

As an immediate consequence, we have: 
Theorem 6.2 The sentence

$C s t b \mathrm{R} \Rightarrow B I_{G}$

is valid over game models. In English: (if we assume common knowledge of the structure of the game and of open future, then) common stable true belief in (dynamic) rationality implies the backward induction outcome.

\section{Comparison with other work}

The game-theoretic issues that we deal with in this paper originate in the work of Aumann (1995), Stalnaker (1994, 1996, 1998) and Reny (1992), and have been investigated by a number of authors Binmore (1987), Binmore (1996), Bicchieri (1989), Battigalli (1997), Battigalli and Siniscalchi (1999, 2002), Bonanno (1991), Brandenburger (2007), Halpern (2001), Samet (1996), Clausing (2003) etc. Our work obviously owes a great deal to these authors for their illuminating discussions of the topic.

The logic CDL of conditional belief was first introduced and axiomatised by Board (2002), in a slightly more complicated form. The version presented here is due to Baltag and Smets (2006, 2008b). The dynamic extension of CDL obtained by adding the public announcements modalities (coming from the public announcement logic PAL, originally developed by Plaza (1989)) has been developed by van Benthem (2007a) and, independently, by Baltag and Smets (2006). The extension of PAL with arbitrary announcement modalities [!] $\varphi$ is due to Balbiani et al. (2008). The beliefrevision-friendly version of APAL presented here (obtained by combining APAL with $\mathrm{CDL}$ ) is an original contribution of our paper.

The work of Battigalli and Siniscalchi (2002) is the closest to ours, both through their choice of the basic setting for the "static logic" (also given by conditional belief operators) and through the introduction of a strengthened form of common belief ("common strong belief") as an epistemic basis for a backward-induction theorem. Strong belief, though different from our "stable" belief, is another version of persistent belief: belief that continues to be maintained unless and until it is contradicted by new information. However, their notion of rationality is only "partially dynamic": although taking into account the dynamics of beliefs (using conditional beliefs given node $v$ to assess the rationality of players' choices at $v$ ), it does not fully take into account the limitations posed to the set of possible options by the dynamics of "hard knowledge". In common with most other previous notions of rationality, it requires agents to make rational choices at all nodes, including the past ones and the ones that have already been bypassed. As a result, it is enough for a player to make only one "irrational" move to completely shatter the (common) belief (however strong) in rationality; and as a consequence, common strong belief in rationality does not by itself imply backward induction. To obtain their theorem, Battigalli and Siniscalchi have to add another assumption: that the game model is a complete type structure, i.e. it contains, in a certain sense, every possible epistemic-doxastic "type" for each player. This means that the players are assumed to have absolutely no "hard" information, not only about the outcomes or about the other players' strategies, but also about the other players' 
beliefs, so that they have to consider as epistemically possible all consistent (probabilistic) belief assignments for the other players! This is an extremely strong (and, in our opinion, unrealistic) "completeness" assumption, one that can only be fulfilled in an infinite model. In contrast, the analogue completeness assumption in our approach is the much weaker "Open Future" assumption, postulating that (at the beginning of the game) players have no non-trivial "hard" information about the outcomes (except the information given by the structure of the game): they cannot foretell the future, cannot irrevocably know the players' freely chosen future moves (though they do irrevocably know the past, and they may irrevocably know the present, including all the beliefs and the plans of action of all the players). Our more realistic postulate is weak enough to be realized on finite models. In particular, it can be realized on models as small as the set of terminal nodes of the game tree (having one state for each terminal node), and in which all the plans of action are common knowledge, so that the only uncertainty concerns possible mistakes in playing (and hence the final outcome).

Samet (1996) introduces a notion of hypothetical knowledge, in order to develop an epistemic characterisation of backward induction. Hypothetical knowledge looks prima facie similar to conditional belief, except that the interpretation of the hypothetical knowledge formula $K_{i}^{\varphi} \psi$ is different: "Had $\varphi$ been the case, $i$ would have known $\psi$ " (Samet 1996, p. 237). This mixture of counterfactual conditionals and knowledge is specifically introduced in Samet (1996) only to discuss backward induction, and it has not occurred before or subsequently in the literature. In contrast, our approach is grounded in the relatively standard and well-understood foundations of Conditional Doxastic Logic, independently studied by logicians and philosophers. While Samet does make what we agree is the important point that some form of counterfactual reasoning is of vital importance to the epistemic situation in extensive games, his model and conditions seem to us more complex, less transparent and less intuitive than ours.

We are aware of only one prior work that uses dynamic epistemic logic (more precisely, the logic of public announcements, but in the context of "classical DEL", i.e. dealing only with knowledge update and not with belief revision) for the analysis of solution concepts in extensive games: van Benthem (2007b). That work takes Aumann's "static" notion of rationality as given, and accepts Aumann's classical result as valid, and so it does not attempt to deal with the cases in which Aumann's assumptions do not apply, nor to address the criticism and the issues raised by Stalnaker, Reny and others. Instead, van Benthem's contribution focuses on the sources of knowledge, on explaining how complex epistemic conditions of relevance to Game Theory (such as Aumann's common knowledge of rationality) can be brought about, via repeated public announcements of rationality. So van Benthem does not use public announcements in order to simulate a play of the game. Public announcements in van Benthem's approach represent off-line learning, i.e. pre-play or inter-play learning, whereas the public announcements in our present approach simulate on-line learning, i.e. learning that takes place during the play of the game. A very interesting open question is to address the same issue answered by van Benthem, but for the case of the dynamicepistemic condition proposed here, instead of Aumann's condition: find some off-line communication or learning protocol that can achieve common knowledge of stable common belief in rational play. 
We should say how our result resolves the apparently conflicting positions of Aumann and Stalnaker. Under sympathetic interpretations of those authors, we would say that we agree with both of them:

We have already commented on the differences between our approach and that of Aumann (1995). However, in order to find a similarity, notice that if we say that strategies are beliefs, then the condition we give begins to look a little like common knowledge of rational strategies. (This identification of strategies with beliefs was not possible in Aumann's framework, so even from this perspective our work would be a considerable advance.)

Stalnaker writes that "[t]he rationality of choices in a game depend[s] not only on what players believe, but also on their policies for revising their beliefs" (1998, p. 31). He then gives a condition on belief revision policies in terms of "epistemic independence" of the players. We agree entirely with the sentiment in the quoted sentence. Indeed game models provide a specification of exactly how players will revise their beliefs, including their beliefs about other players' beliefs, so that these beliefs remain consistent no matter how the play of the game goes. Theorem 4.4 goes further and specifies conditions necessary on such models, purely in terms of epistemic and doxastic attitudes towards rationality, that ensure the backward induction outcome. Stable belief in dynamic rationality is in effect a partial description of an "optimistic" belief revision policy, that says: "when you revise your beliefs, maintain at all costs a belief in the opponents' rational potential, despite their past deviations from rationality'.

In fact, as mentioned in the introduction, whether this policy can appropriately be called "optimistic" or "pessimistic" depends on the game and the players' payoffs. In many contexts, such an "incurably optimistic" (or "persistently pessimistic") revision policy may seem naïve, but our point is that only such a policy can offer a rational doxastic justification to backward induction. The well-known examples of 'catastrophic' BI outcomes can thus been seen to illustrate the dangers of "rational" pessimism, while the examples of 'desirable' BI outcomes illustrate the saving power of "incurable" optimism.

Acknowledgements The authors would like to give special thanks to Johan van Benthem for his insights and the stimulating discussions that lead to this paper. The last two authors would also like to thank Larry Moss for his help, including for organising the Bloomington workshop at which a draft of this paper was first presented. We are grateful to the two anonymous referees for their comments. The research of the first author was partially supported by the Netherlands Organisation for Scientific Research (NWO), grant number B 62-635, which is herewith gratefully acknowledged. The second author acknowledges the support by the Flemish Fund for Scientific Research in the early stage of this paper and support by the University of Groningen via a Rosalind Franklin research position in the last stage of this paper. The last author was supported by a Marie Curie Early Stage Research fellowship in the project GLoRiClass (MEST-CT-2005-020841).

Open Access This article is distributed under the terms of the Creative Commons Attribution Noncommercial License which permits any noncommercial use, distribution, and reproduction in any medium, provided the original author(s) and source are credited.

\section{References}

Alchourrón, C. E., Gärdenfors, P., \& Makinson, D. (1985). On the logic of theory change: Partial meet contraction and revision functions. Journal of Symbolic Logic, 50(2), 510-530. 
Aumann, R. (1995). Backward induction and common knowledge of rationality. Games and Economic Behavior, 8, 6-19.

Balbiani, P., Baltag, A., van Ditmarsch, H., Herzig, A., Hoshi, T., \& de Lima, T. (2008). 'Knowable' as 'known after an announcement'. The Review of Symbolic Logic, 1(3), 305-334. Published online by Cambridge University Press, December 15, 2008. doi:10.1017/S1755020308080210.

Baltag, A., \& Smets, S. (2006). Conditional doxastic models: A qualitative approach to dynamic belief revision. Electronic Notes in Theorerical Computer Science, 165, 5-21.

Baltag, A., \& Smets, S. (2008a). The logic of conditional doxastic actions. In R. van Rooij \& K. R. Apt (Eds.), New perspectives on games and interaction, Vol. 4 of Texts in logic and games (pp. 9-31). Amsterdam: Amsterdam University Press.

Baltag, A., \& Smets, S. (2008b). A qualitative theory of dynamic interactive belief revision. In G. Bonanno, W. v. d. Hoek \& M. Wooldridge (Eds.), Logic and the Foundations of Game and Decision Theory (LOFT 7), Vol. 3 of Texts in logic and games. (pp. 9-58). Amsterdam: Amsterdam University Press.

Baltag, A., Moss L. S., \& Solecki, S. (1999). The logic of public announcements, common knowledge and private suspicions. Technical Report SEN-R9922, Centrum voor Wiskunde en Informatica.

Battigalli, P. (1997). On rationalizability in extensive games. Journal of Economic Theory, 74(1), 40-61.

Battigalli, P., \& Siniscalchi, M. (1999). Hierarchies of conditional beliefs and interactive epistemology in dynamic games. Journal of Economic Theory, 88(1), 188-230.

Battigalli, P., \& Siniscalchi, M. (2002). Strong belief and forward induction reasoning. Journal of Economic Theory, 106(2), 356-391.

Bicchieri, C. (1989). Self-refuting theories of strategic interaction: a paradox of common knowledge. Erkenntnis, 30, 69-85.

Binmore, K. (1987). Modeling rational players, part I. Economics and Philosophy, 3, 179-214.

Binmore, K. (1996). A note on backward induction. Games and Economic Behavior, 17(1), 135-137.

Blume, L., Brandenburger, A., \& Dekel, E. (1991). Lexicographic probabilities and equilibrium refinements. Econometrica, 59(1), 81-98.

Board, O. (2002). Dynamic interactive epistemology. Games and Economic Behavior, 49, 49-80.

Bonanno, G. (1991). The logic of rational play in games of perfect information. Economics and Philosophy, 7, 37-65.

Brandenburger, A. (2007). The power of paradox: Some recent developments in interactive epistemology. International Journal of Game Theory, 35(4), 465-492.

Clausing, T. (2003). Doxastic conditions for backward induction. Theory and Decision, 54, 315-336.

Fagin, R., Halpern, J., Moses, Y., \& Vardi, M. (1995). Reasoning about knowledge. Cambridge, MA: MIT Press.

Gerbrandy, J. D., \& Groeneveld, W. (1997). Reasoning about information change. Journal of Logic, Language, and Information, 6, 147-169.

Grove, A. (1988). Two modellings for theory change. Journal of Philosophical Logic, 17(2), 157-170.

Halpern, J. Y. (2001). Substantive rationality and backward induction. Games and Economic Behavior, 37, 425-435.

Moore, G. (1942). A reply to my critics. In P. Schilpp (Ed.), The philosophy of G.E. Moore, Vol. 4 of The library of living philosophers (pp. 535-677). Evanston, IL: Northwestern University.

Osborne, M. J., \& Rubinstein, A. (1994). A course in game theory. Cambridge, MA: MIT Press.

Plaza, J. A. (1989). Logics of public communications. In M. L. Emrich, M. S. Pfeifer, M. Hadzikadic \& Z. W. Ras (Eds.), Proceedings of the 4th international symposium on methodologies for intelligent systems (pp. 201-216).

Rabinowicz, W. (1998). Grappling with the centipede: defense of backward induction for BI-terminating games. Philosophy and Economics, 14, 95-126.

Reny, P. (1992). Rationality in extensive form games. Journal of Economic Perspectives, 6, 92-100.

Rényi, A. (1955). On a new axiomatic theory of probability. Acta Mathematica Academiae Scientiarum Hungaricae, 6, 285-335.

Rosenthal, R. (1981). Games of perfect information, predatory pricing, and the chain store. Journal of Economic Theory, 25, 92-100.

Roy, O. (2008). Thinking before acting: intentions, logic, rational choice. Ph.D. thesis, ILLC, Amsterdam.

Samet, D. (1996). Hypothetical knowledge and games with perfect information. Games and Economic Behavior, 17, 230-251.

Stalnaker, R. C. (1994). On the evaluation of solution concepts. Theory and Decision, 37, 49-73. 
Stalnaker, R. C. (1996). Knowledge, beliefs and counterfactual reasoning in games. Economics and Philosophy, 12, 133-163.

Stalnaker, R. C. (1998). Belief revision in games: Forward and backward induction. Mathematical Social Sciences, 36, 31-56.

Stalnaker, R. C. (2006). On logics of knowledge and belief. Philosophical Studies, 128, 169-199.

van Benthem, J. (2007a). Dynamic logic for belief revision. Journal of Applied Non-Classical Logics, 17(2), $129-155$.

van Benthem, J. (2007b). Rational dynamics and epistemic logic in games. International Game Theory Review, 9(1), 13-45. (Erratum reprint, 9(2), 377-409). 